Dr. Christian Honert ist neuer Koordinator der Initiative „young dentists“

\title{
„Ich möchte junge Kollegen auf die Zeit der Niederlassung vorbereiten"
}

\author{
Den „young dentists“ ist Dr. Christian Honert schon lange treu. Seit November vergangenen Jah- \\ res ist der 40-jährige niedergelassene Zahnarzt aus Asbach Koordinator der Initiative. Warum er \\ sich auf die Arbeit freut, hat er der DFZ-Redaktion verraten.
}

DFZ: Herr Honert, wie lange sind Sie schon bei „young dentists“ aktiv?

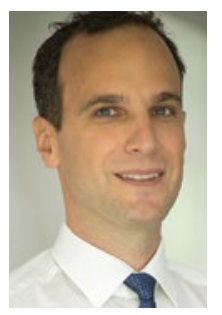

Honert: Schon ziemlich lange. Ich war 2008 das erste Mal als junger Zahnarzt bei einer Fortbildung der Initiative. Und dann bin ich, salopp gesagt, kleben geblieben. Weil mir die Fortbildungen und später dann auch die regelmäßigen „young dentists meetings" sehr gut gefallen haben, habe ich immer wieder teilgenommen und bin Mitglied des Beirates geworden, in dem ich an der Auswahl der Themen für die Treffen und anderem beteiligt war. Und Ende vergangenen Jahres fragte mich Dr. Norbert Grosse dann, ob ich sein Nachfolger als Koordinator der Initiative werden möchte. Das hat mich sehr gefreut, und ich habe sofort zugesagt.

DFZ: Was ist den das Tolle an „young dentists"?

Honert: Ganz klar die Atmosphäre. Bei den „young dentists meetings "in Bonn trifft man junge angehende Kolleginnen und Kollegen, die in derselben Situation sind wie man selbst und die ähnliche Fragen mitbringen. Das Treffen ist immer sehr familiär. Wer einmal da war, kommt immer wieder.

Das Besondere an der Fortbildung ist die Kombination aus Fachlichem und dem entspannten Zusammensein zwischen und nach den Seminaren. Da sitzen abends die Referenten mit beim Essen, und jeder kann individuelle Fragen stellen. Das ganze Paket stimmt einfach. Statt Massenfortbildung mit Frontalunterricht setzen wir auf den Dialog und Hands-on-Kurse, in denen jeder auf seine Kosten kommt.

DFZ: Wer darf ein „young dentist“ werden und an den Treffen teilnehmen?

Honert: Wir haben derzeit 1.400 angemeldete „young dentists“. Die Mitgliedschaft ist kostenlos. Mitglied werden können Studenten, Assistenten und Praxisgründer bis fünf Jahre nach der Niederlassung. Es sind alle willkommen.

DFZ: Wollen Sie an dem bisherigen Konzept der „young dentists meetings" etwas ändern?

Honert: Nein, das Konzept hat sich bewährt. Wir starten immer freitags mit einem ökonomisch-praktischen Teil. Dort steht ein Thema auf dem Programm, das während des Studiums nicht beleuchtet wird, aber im Praxisalltag von entscheidender Bedeutung ist.
Und am zweiten Tag gibt es einen fachlichen, zahnmedizinischen Teil. Die Mischung ist gut und gefällt den Teilnehmern.

DFZ: Und wenn es mal nicht gefällt, gibt es dann Änderungen? Honert: Ich bin für alles offen. Nach jedem Treffen befragen wir die Teilnehmer, wie sie es fanden. Und dann werten wir die Evaluationsbögen aus. Falls Änderungswünsche bestehen, nehmen wir diese gerne auf und versuchen, sie zu realisieren. Wir sind, was die Themen angeht, sehr flexibel.

DFZ: Sie sagen, das Konzept von „young dentists“ ist etwas Besonderes. Gibt es für junge Zahnärzte in Deutschland nichts Vergleichbares?

Honert: Nein, ich weiß jedenfalls von nichts Vergleichbarem.

DFZ: Was ist für Sie persönlich der Reiz an der neuen Aufgabe des Koordinators?

Honert: Ich finde es sehr spannend, mit jungen Leuten zusammenzuarbeiten. Und mir liegt die Freiberuflichkeit am Herzen. Ich möchte junge Kollegen auf die Zeit der Niederlassung vorbereiten, die durchaus den einen oder anderen Stolperstein birgt. Außerdem möchte ich die „young dentists“ dabei unterstützen, Netzwerke zu bilden, damit sie ihre Erfahrungen austauschen können und voneinander profitieren. Es ist gerade als Berufsanfänger hilfreich, wenn man mit Problemen nicht alleine klarkommen muss.

Interview: Melanie Fügner Alle Infos zum nächsten „young dentists meeting“ gibt es auf Seite 70.

young dentists: Orientierungshilfe für Berufseinsteiger

Die Initiative young dentists ist eine Kooperation zwischen dem Freien Verband Deutscher Zahnärzte (FVDZ) und der Akademie und Wissenschaft (APW) in der Deutschen Gesellschaft für Zahn-, Mund- und Kieferheilkunde (DGZMK). Diese gemeinsame Initiative bietet dem zahnärztlichen Nachwuchs Orientierungshilfe und Beistand in den ersten Jahren des Berufsweges, bringt junge Zahnmedizinerinnen und Zahnmediziner zusammen und zeigt Wege auf, erfolgreich die Karriere zu gestalten. Zweimal im Jahr treffen sich Interessierte beim zweitägigen „young dentists meeting" in Bonn. 